\title{
Cut-Elimination Theorem Concerning a Formal System for Ramified Theory of Types Which Admits Quantifications on Types
}

\author{
Shôji MaEhara \\ Waseda University, Tokyo
}

In 1938, Prof. K. Ono introduced a ramified theory of types, and he proved the consistency of a formal number theory with restricted induction schema ${ }^{1)}$ by reducing the consistency of the latter theory to that of the former (cf. Ono [3]).

In fact, he did not formulate the theory of types oneself as a formal system, but he stated methods which were named 'Erweiterungsprinzipien' and by which, when an axiom system formalized in the first-order predicate calculus is given, we can develope, in the scope of the first-order predicate calculus, various theories of types based on the given axiom system.

The purpose of this paper is to formulate a ramified theory of types as a formal system, and to prove the cut-elimination theorem on that system. If an axiom system consisting of only formulae in the first-order predicate calculus is treated in that formal system, the formal theory which was named ' $\mathbf{M}^{n} \mathbf{P}$-Erweiterung' of the axiom system in Ono [3], and which is a ramified theory of types based on the axiom system, will be essentially contained in that treatment, for every natural number $n$. And, as an application, we shall give a modification of Ono's consistency proof for formal number theory with restricted induction schema. In author's opinion, by this modification the essence of Prof. Ono's idea for the consistency proof will be made more clear.

\section{§1. Formal system for ramified theory of types.}

In our formal system for ramified theory of types, we use predicate variables of type $\alpha$, for every type $\alpha$. If $a$ means an element of the domain of individuals and $F_{\alpha}$ is a predicate variable of type $\alpha$, then $F_{\alpha}(a)$ means informally that $a$ has the property $F_{\alpha}$ of type $\alpha$, or that $a$ belongs to the set $F_{\alpha}$ of type $\alpha$. In this sense, predicate variables mean predicates of first order and with one argument. That we use no predicate variables of higher order nor predicate variables with several arguments is because of making our descriptions simple and of making the point of our argument clear.

As types of predicate variables we use arbitrary ordinal numbers less than $\omega^{(1)}$, and some expressions representing such an indefinite ordinal numbers. Variables

1) In Prof. Ono's opinion, almost all mathematical inductions which are practically used in the number theory are said to be formulated in this restricted form. 
contained in the type of a predicate variable, if there exist, are only variables for finite ordinal numbers (=non-negative integers).

1. Exact definition of the concept 'type'.

1.1. DEFINITION of ' $n$-term':

1.11. 0 is an $n$-term.

1.12. Each of the free $n$-variables

$$
n, n_{0}, n_{1}, n_{2}, \cdots
$$

is an $n$-term. (Informally, $n$-variables are variables for non-negative integers.)

1.13. If $\mathfrak{m}$ is an $n$-term, then $\mathfrak{m}+1$ is an $n$-term. If $\mathfrak{m}_{1}$ and $\mathfrak{m}_{2}$ are $n$-terms, then $\max \left\{\mathfrak{m}_{1}, \mathfrak{m}_{2}\right\}$ is an $n$-term. (Informally, $\max \left\{\mathfrak{m}_{1}, \mathfrak{m}_{2}\right\}$ means the greatest number of $\mathfrak{m}_{1}$ and $\mathfrak{m}_{2}$.)

1.14. The only $n$-terms are those given by $1.11,1.12$ and 1.13 .

1.2. Definition of 'type'. A type is an expression of the form

$$
\omega^{\nu} \mathfrak{m}_{\nu}+\omega^{\nu-1} \mathfrak{m}_{\nu-1}+\cdots+\omega \mathfrak{m}_{1}+\mathfrak{m}_{0},
$$

where $\nu$ is a non-negative integer and $\mathfrak{m}_{0}, \mathfrak{m}_{1}, \cdots, \mathfrak{m}_{\nu}$ are arbitrary $n$-terms.

1.3. Types are formal expressions, but, for the sake of simplification of notations, we shall also use usual expressions, for example, as stated below.

Example. By

$$
\omega^{3}(n+2)+\omega
$$

we mean the type

$$
\omega^{3}((n+1)+1)+\omega^{2} 0+\omega(0+1)+0 .
$$

2. 'Formula'.

2.1. Additional primitive symbols :

2.11. Free predicate variables of type $\alpha: F_{\alpha}, G_{\alpha}, \cdots$, for every type $\alpha$.

2.12. Bound predicate variables of type $\alpha: P_{\alpha}, Q_{\alpha}, \cdots$, for every type $\alpha$.

2.13. Bound $n$-variables: $l, l_{0}, l_{1}, l_{2}, \cdots$.

2.2. When a type $\alpha$ is given, we define the concept 'formula of type $\alpha$ ', as follows :

2.21. Formulae in the sense of the first-order predicate calculus are formulae of type $\alpha$.

2.22. If $F_{\beta}$ is an arbitrary free predicate variable of type $\beta$ and $t$ is a term in an ordinary sense ${ }^{2}$, and if the inequality $\alpha \geqq \beta$ is always true in an ordinary sense, then $F_{\beta}(t)$ is a formula of type $\alpha$.

2.23. If $\mathfrak{A}$ is a formula of type $\alpha$, then $>\mathfrak{A}$ is a formula of type $\alpha$. If $\mathfrak{A}$ and $\mathfrak{B}$ are formulae of type $\alpha$, then $\mathfrak{A} \& \mathfrak{B}, \mathfrak{A} \vee \mathfrak{B}$ and $\mathfrak{A} \supset \mathfrak{B}$ are formulae of type $\alpha$. If $\mathfrak{A}(a)$ is a formula of type $\alpha, a$ is a free individual variable, and $x$ is an arbitrary bound individual variable not contained in $\mathfrak{A}(a)$, then $\forall x \mathfrak{I}(x)$ and $\exists x \mathfrak{H}(x)$ are formulae of type $\alpha$, where $\mathfrak{A}(x)$ means the result of substituting $x$ for $a$ throughout $\mathfrak{A}(a)$.

2.24. If $\mathfrak{A}\left(F_{\beta}\right)$ is a formula of type $\alpha, F_{\beta}$ is a free predicate variable of type

2) English terminologies on the predicate calculus will be used, for most part, according to the usage in Kleene's text book [2]. 
$\beta$, and $P_{\beta}$ is an arbitrary bound predicate variable of type $\beta$ not contained in $\mathscr{U}\left(F_{\beta}\right)$, and if the inequality $\alpha>\beta$ is always true in an ordinary sense, then $\forall P_{\beta} \mathfrak{A}\left(P_{\beta}\right)$ and ${ }_{3} P_{\beta} \mathfrak{Y}\left(P_{\beta}\right)$ are formulae of type $\alpha$, where $\mathfrak{X}\left(P_{\beta}\right)$ means the result of substituting $P_{\beta}$ for $F_{\beta}$ throughout $\mathfrak{A}\left(F_{\beta}\right)$.

2.25. If $\mathfrak{M}(n)$ is a formula of type $\alpha, n$ is a free $n$-variable not contained in $\alpha$, and $l$ is an arbitrary bound $n$-variable not contained in $\mathfrak{A}(n)$, then $\forall l \mathfrak{A}(l)$ and $\exists l \mathfrak{A}(l)$ are formulae of type $\alpha$, where $\mathfrak{A}(l)$ means the result of substituting $l$ for $n$ throughout $\mathfrak{A}(n)$.

2.26. The only formulae of type $\alpha$ are those given by 2.21-2.25.

2.3. If $\mathfrak{A}$ is a formula of type $\alpha$, then $\alpha$ is called a type of the formula $a^{3)}$.

3. 'Sequent'.

A sequent is a formal expression of the form

$$
\mathfrak{A}_{1}, \mathfrak{A}_{2}, \cdots, \mathfrak{A}_{\mu} \rightarrow \mathfrak{B}_{1}, \mathfrak{B}_{2}, \cdots, \mathfrak{B}_{\nu},
$$

where $\mu, \nu \geqq 0$ and $\mathfrak{A}_{1}, \mathfrak{A}_{2}, \cdots, \mathfrak{A}_{\mu}, \mathfrak{B}_{1}, \mathfrak{B}_{2}, \cdots, \mathfrak{B}_{\nu}$ are arbitrary formulae.

4. 'Rules of inference'.

As rules of inference we use ones for Gentzen's $L K$, which are represented as the 'Schlußfiguren-Schemata', as they are, provided that the letters expressing a formula or a sequence of formulae may be substituted by an arbitrary formula in our sense or an arbitrary finite sequence of them, and the follwing.

Additional rules of inference.

Introduction of in antecedent. in succedent.
$\mathrm{A} P_{\alpha}:$
$\frac{\mathfrak{A}(\lambda x \mathfrak{F}(x)), \Gamma \rightarrow \Theta}{\forall P_{\alpha} \mathfrak{A}\left(P_{\alpha}\right), \Gamma \rightarrow \Theta}$
$\frac{\Gamma \rightarrow \Theta, \mathfrak{A}\left(F_{\alpha}\right)}{\Gamma \rightarrow \Theta, \forall P_{\alpha} \mathfrak{A}\left(P_{\alpha}\right)}$
${ }_{\exists} P_{\alpha}:$
$\frac{\mathfrak{A}\left(F_{\alpha}\right), \Gamma \rightarrow \Theta}{\exists P_{\alpha} \mathfrak{A}\left(P_{\alpha}\right), \Gamma \rightarrow \Theta}$
$\frac{\Gamma \rightarrow \Theta, \mathfrak{A}(\lambda x(\mathfrak{F} x))}{\Gamma \rightarrow \Theta,{ }^{\prime} P_{\alpha} \mathfrak{I}\left(P_{\alpha}\right)}$
$\forall l:$
$\frac{\mathfrak{A}(\mathfrak{m}), \Gamma \rightarrow \Theta}{\forall l \mathfrak{A}(l), \Gamma \rightarrow \Theta}$
$\frac{\Gamma \rightarrow \Theta, \mathfrak{U}(n)}{\Gamma \rightarrow \Theta, \forall l \mathfrak{A}(l)}$
$\exists l:$

$$
\frac{\mathfrak{A}(n), \Gamma \rightarrow \Theta}{\exists l \mathfrak{A}(l), \Gamma \rightarrow \Theta}
$$$$
\frac{\Gamma \rightarrow \Theta, \mathfrak{Y}(\mathrm{m})}{I \rightarrow \Theta, \exists l \mathfrak{X}(l)}
$$

STIPULATIONS :

4.1. $\Gamma$ and $\Theta$ are arbitrary finite sequences of zero or more formulae.

4.2. $\forall P_{\alpha} \mathfrak{I}\left(P_{\alpha}\right)$ or $\exists P_{\alpha} \mathfrak{I}\left(P_{\alpha}\right)$ is an arbitrary formula of such a form, where $P_{\alpha}$ is an arbitrary bound predicate variable of type $\alpha$. And at this time $\mathfrak{A}(\lambda x \mathfrak{F}(x))$ or $\mathfrak{A}\left(F_{\alpha}\right)$ is the result of substituting $\lambda x \mathfrak{F}(x)$ or $F_{\alpha}$ for $P_{\alpha}$ throughout $\mathfrak{A}\left(P_{\alpha}\right)$. For an arbitrary given formula $\mathfrak{F}(a)$ with a free individual variable $a$, the formula $\mathfrak{A}(\lambda x \mathfrak{F}(x))$ can be exactly defined without using the $\lambda$-symbol.4)

4.3. In an application of the 'introduction of $\forall P_{\alpha}$ in antecedent' or 'introduction of $\exists P_{\alpha}$ in succedent', $\widetilde{\mho}(a)$ must be a formula of type $\alpha$.

4.4. In an application of the 'introduction of $\forall P_{\alpha}$ in succedent' or 'introduc-

3) The concept of formula which is defined here corresponds to 'gesonderte Formel' in the sense of Ono [3].

4) The expression $\{\lambda x \mathfrak{F}(x)\}(\mathfrak{t})$ means $\mathfrak{F}(\mathrm{t})$. 
tion of $\exists P_{\alpha}$ in antecedent', $F_{\alpha}$ is a free predicate variable of type $\alpha$ which is arbitrarily chosen but is never contained in the conclusion.

4.5. $\forall \mathfrak{I}(l)$ or $\exists l \mathfrak{A}(l)$ is an arbitrary formula of such a form, where $l$ is an arbitrary bound $n$-variable. And at this time $\mathfrak{A}(\mathfrak{m})$ and $\mathfrak{A}(n)$ is the result of substituting an arbitrary $n$-term $\mathfrak{m}$ or an arbitrary free $n$-variable $n$ for $l$ throughout $\mathfrak{U}(l)$.

4.6. In an application of the 'introduction of $\forall l$ in succedent' or 'introductin of $\exists l$ in antecedent', the free $n$-variable $n$ will never occur in the conclusion.

5. 'Proof'.

As formal proofs we use only ones in tree form, each of which has one lowermost sequent-the endsequent-and some uppermost sequents of the form

$$
\mathfrak{D} \rightarrow \mathfrak{D} \text {, }
$$

where $\mathscr{D}$ is an arbitrary formula.

A proof of a sequent is a formal proof which has the sequent as the endsequent. A sequent is said to be provable, if there exists a proof of the sequent. A sequent is said to be provable without cut, if there exists a proof of the sequent which contains no inference conforming the rule

Cut :

$$
\frac{\Gamma \rightarrow \Theta, \mathfrak{D} \mathscr{D}, \Delta \rightarrow A}{\Gamma, \Delta \rightarrow \Theta, A}
$$

named 'Schnitt' in Gentzen [1].

\section{§2. Cut-elimination theorem.}

The fundamental theorem of our formal system for ramified theory of types is the following

CUT-eLIMINATION THEOREM. Any provable sequent is provable without cut.

This theorem corresponds to Gentzen's Hauptsatz on $L K$, and also the proof is carried through in almost the same way as in Gentzen's proof of his Hauptsatz, provided that the concept 'grade of formula' is appropriately defined and, for every inference concerning the introduction of a logical symbol, the grade of the 'Hauptformel' is greater than those of one or two 'Nebenformeln'. Then, in what follows, we shall give only the definition of the concept 'grade of formula'.

Our formula contains, in general, quantifiers of the form $\forall P_{\xi}$ or $\exists P_{\xi}$, where $\xi$ is a type or the result obtained from a type by substituting bound $n$-variables for some free $n$-variables. Now let $\xi$ have the form

$$
\omega^{\nu} p_{\nu}+\omega^{\nu-1} \mathfrak{p}_{\nu-1}+\cdots+\omega p_{1}+p_{0},
$$

where each of p's is an $n$-term or the result obtained from an $n$-term by substituting bound $n$-variables for some free $n$-variables. Then, by $\|\xi\|$ we shall mean the ordinal number

$$
\omega^{2 \nu}\left|p_{\nu}\right|+\omega^{2 \nu-2}\left|p_{\nu-1}\right|+\cdots+\omega^{2}\left|p_{1}\right|+\left|p_{0}\right|
$$

where $|\mathfrak{p}|$ is the result of substituting 0 for all free $n$-variables and $\omega$ for all bound $n$-variables throughout $p$. By the grade of an occurrence of a logical symbol in a given formula, we mean the ordinal number $\omega^{i \xi \|+1}$, if the occurrence is used 
in the form $\forall P_{\xi}$ or $\exists P_{\xi}$, and we mean the ordinal number 1 , if the occurrence is used otherwise. Let $\gamma_{1}, \gamma_{2}, \cdots, \gamma_{\mu}$ be the grade of all occurrence of logical symbols in a given formula and

then the ordinal number

$$
r_{1} \geqq r_{2} \geqq \cdots \geqq r_{\mu},
$$

$$
r_{1}+r_{2}+\cdots+r_{\mu}
$$

is called the grade of the given formula.

The cut-elimination theorem on our formal system can be easily proved by using the transfinite induction on the grade of formula, instead of the mathematical induction on the grade of formula in Gentzen's proof of the Hauptsatz on $L K$ (cf. [1]).

\section{§3. Consistency proof of a number theory with restricted induction schema.}

As is well known, a full system for the pure number theory is obtained from the number theory without mathematical induction by adjoining a new fixed predicate symbol $Z$ of one argument and the axioms

$$
Z(1), \quad \forall x(Z(x) \supset Z(x+1)), \quad \forall x \forall y[x=y \supset(Z(x) \supset Z(y))]
$$

and the axiom schema

$$
\{\mathfrak{U}(1) \& \forall x[Z(x) \supset(\mathfrak{A}(x) \supset \mathfrak{U}(x+1))]\} \supset \forall x(Z(x) \supset \mathfrak{A}(x)) .
$$

What we call here 'number theory with restricted induction schema' is the subsystem obtained from the above-mentioned full system for the pure number theory by restricting the use of the axiom schema $\left(^{*}\right)$ in the following manner.

RESTRICTION ON INDUCTION FORMULAE. The induction formula $\mathfrak{A}(a)$ of the axiom schema (*) must be $Z$-positive or $Z$-negative in the following sense. A formula is said to be $Z$-positive or $Z$-negative, if and only if every occurrence of the predicate symbol $Z$ in the prenex disjunctive normal form of the formula is not preceded or is preceded by the negation symbol, respectively.5) If a formula contains no $Z$, of course, it is not only $Z$-positive but also $Z$-negative.

1. Preliminaries.

1.1. Firstly, we shall extend the concept 'formal proof' in the ramified theory of types which has been formulated in $\S 1$, as follows:

As the uppermost sequents of a formal proof, we may use any sequent containing no logical symbol, provided that it is effectively true in the sense of the usual interpretation.

The cosistency of this extended system can be easily proved, by help of the cut-elimination theorem on the original system, or by proving directly the cutelimination theorem on the extended system.

Secondly, we shall only use arbitrary number-theoretic predicates and functions, each of which is effectively decidable or calculable, as the predicate and function symbols, except the predicate variables.

In what follows, this modified system will be called simply 'ramified theory'.

5) In Ono [3], such a formula is called 'einseitige Formel in bezug auf $Z$ '. 
1.2. Definition. For each type $\alpha$, by $3(\alpha, a)$ we mean the formula

$$
\forall P_{\alpha}\left\{\left[P_{\alpha}(1) \& \forall x\left(P_{\alpha}(x) \supset P_{\alpha}(x+1)\right)\right] \supset \exists y\left(\alpha=y \& P_{\alpha}(y)\right)\right\} \text {. }
$$

THEOREM 1. Let $\alpha$ and $\beta$ be types, and $\xi$ and $\mathrm{t}$ be terms, then the following sequents are provable in the ramified theory:

1) $\mathfrak{Z}(\alpha, \mathrm{f}) \rightarrow \mathcal{Z}(\beta, 1)$,

where the inequality $\alpha>\beta$ is always true;

2) $\rightarrow \boldsymbol{Z}(\alpha, 1)$;

3) $3(\alpha, \mathfrak{t}) \rightarrow 3(\alpha, \mathfrak{t}+1)$;

4) $\mathfrak{z}=\mathfrak{\ddagger}, \mathfrak{Z}(\alpha, \underline{g}) \rightarrow \mathfrak{Z}(\alpha, t)$;

5) $3(\alpha, t+1) \rightarrow 3(\alpha, t)$.

Proof. 1)-4) are easily proved. We shall prove only 5).

Let $\mathfrak{A}(a)$ mean the formula

$$
P_{\alpha}(a) \& \forall x\left[x+1=a \supset \exists y\left(x=y \& P_{\alpha}(y)\right)\right] .
$$

Then the sequents

and

$$
P_{\alpha}(1) \rightarrow \mathfrak{A}(1)
$$

$$
P_{\alpha}(a) \supset P_{\alpha}(a+1), \mathfrak{A}(a) \rightarrow \mathfrak{A}(a+1)
$$

are provable. Hence, by the definition of 3 , the sequent

$$
P_{\alpha}(1), \forall x\left(P_{\alpha}(x) \supset P_{\alpha}(x+1)\right), \mathfrak{Z}(\alpha, t+1) \rightarrow \exists u(t+1=u \& \mathfrak{U}(u))
$$

is provable, accordingly so is the sequent

$$
P_{\alpha}(1), \forall x\left(P_{\alpha}(x) \supset P_{\alpha}(x+1)\right), 3(\alpha, 1+1) \rightarrow \exists y\left(t=y \& P_{\alpha}(y)\right) .
$$

Therefore the sequent

$$
3(\alpha, \mathfrak{t}+1) \rightarrow 3(\alpha, \mathfrak{t})
$$

is provable, q.e.d.

Definition. A formula $\mathfrak{A}(a)$ with a free individual variable $a$ is said to satisfy the equality axiom, if and only if the sequent

$$
\rightarrow \forall x \forall y[x=y \supset(\mathfrak{X}(x) \supset \mathfrak{U}(y))]
$$

is provable.

THEOREM 2. Let $\alpha$ and $\beta$ be types, $\mathfrak{2}(a)$ and $\forall l \mathfrak{Z}\left(\alpha+\omega^{\mu} l, a\right)$ be formulae of type $\beta$, and $\Gamma$ and $\Theta$ be sequences of formulae containing no occurrence of $a$. If the sequents

$$
\begin{aligned}
& \Gamma \rightarrow \Theta, \mathfrak{H}(1), \\
& \forall l \mathfrak{B}\left(\alpha+\omega^{\mu} l, a\right), \mathfrak{A}(a), \Gamma \rightarrow \Theta, \mathfrak{U}(a+1), \\
& \mathfrak{A}(\mathrm{t}), \Gamma \rightarrow \Theta
\end{aligned}
$$

are provable, and if $\mathfrak{U}(a)$ satisfies the equality axiom, then the sequent

$$
\mathfrak{3}(\beta, \mathrm{t}), \Gamma \rightarrow \Theta
$$

is provable.

Proof. By the provability of the former three sequents and by 4) and 5) of Theorem 1, we can see that the sequents 


$$
\begin{aligned}
& \Gamma \rightarrow \Theta, \forall l \mathfrak{Z}\left(\alpha+\omega^{\mu} l, 1\right) \supset \mathfrak{A}(1), \\
& \forall l \mathfrak{B}\left(\alpha+\omega^{\mu} l, a\right) \supset \mathfrak{U}(a), \Gamma \rightarrow \Theta, \forall l \mathbb{Z}\left(\alpha+\omega^{\mu} l, a+1\right) \supset \mathfrak{A}(a+1), \\
& \exists u\left[\mathrm{t}=u \&\left(\forall l \mathfrak{B}\left(\alpha+\omega^{\mu} l, u\right) \supset \mathfrak{A}(u)\right)\right], \forall l \mathfrak{Z}\left(\alpha+\omega^{\mu} l, \mathfrak{t}\right), \Gamma \rightarrow \Theta
\end{aligned}
$$

are provable, and accordingly, by the definition of $3(\beta, t)$, we can see that the sequent

is provable, q.e.d.

$$
3(\beta, \mathrm{t}), \Gamma \rightarrow \Theta
$$

DEFINITION. By $\mathfrak{A}^{*}(\alpha, a)$ we mean the result of substituting $\lambda z \mathfrak{3}(\alpha, z)$ for $Z$ throughout a given formula $\mathfrak{A}(a)$.

THEOREM 3. Let $\alpha, \beta$ and $\forall l\left(3\left(\alpha+\omega^{\mu} l, a\right)\right.$ have the same meaning as in Theorem 2 , and $a$ and $n$ be $a$ free individual variable and a free $n$-variable, respectively, which are contained in neither $\Gamma$ nor $\Theta$. And let $\mathfrak{A}(a)$ be a formula in the sense of the first-order predicate calculus, and contain only decidable number-theoretic predicates and calculable number-theoretic functions, as predicate and function symbols, except the predicate symbol $Z$.

I) If the sequents

$$
\begin{aligned}
& \Gamma \rightarrow \Theta, \mathfrak{P}^{*}\left(\alpha+\omega^{\mu} n, 1\right), \\
& \mathbb{3}\left(\alpha+\omega^{\mu}(n+1), a\right), \mathfrak{2} *\left(\alpha+\omega^{\mu}(n+1), a\right), \Gamma \rightarrow \Theta, \mathfrak{P}^{*}\left(\alpha+\omega^{\mu} n, a+1\right), \\
& \mathfrak{X}^{*}\left(\alpha+\omega^{\mu}(n+1), \mathfrak{t}\right), \Gamma \rightarrow \Theta
\end{aligned}
$$

are provable, then so is the sequent

II) If the sequents

$$
3(\beta, 1), \Gamma \rightarrow \Theta .
$$

$$
\begin{aligned}
& \Gamma \rightarrow \Theta, \mathfrak{A}^{*}\left(\alpha+\omega^{\mu}(n+1), 1\right), \\
& \mathfrak{3}\left(\alpha+\omega^{\mu}(n+1), a\right), \mathfrak{U}^{*}\left(\alpha+\omega^{\mu} n, a\right), \Gamma \rightarrow \Theta, \mathfrak{U}^{*}\left(\alpha+\omega^{\mu}(n+1), a+1\right), \\
& \mathfrak{U}^{*}\left(\alpha+\omega^{\mu} n, \mathfrak{t}\right), \Gamma \leftarrow \Theta
\end{aligned}
$$

are provable, then so is the sequent

$$
3(\beta, \mathfrak{t}), \Gamma \rightarrow \Theta .
$$

Proof. First of all, let us remark the fact that the formulae $\forall l \mathfrak{R}^{*}\left(\alpha+\omega^{\mu} l, a\right)$ and $\exists l \mathfrak{P}^{*}\left(\alpha+\omega^{\mu} l, a\right)$ satisfy the equality axiom, because of 4$)$ of Theorem 1 .

I) By the premises of the theorem, we can see that the sequents

$$
\begin{aligned}
& \Gamma \rightarrow \Theta, \forall l \mathfrak{U}^{*}\left(\alpha+\omega^{\mu} l, 1\right), \\
& \forall l \mathfrak{Z}\left(\alpha+\omega^{\mu} l, a\right), \forall l \mathfrak{A}^{*}\left(\alpha+\omega^{\mu} l, a\right), \Gamma \rightarrow \Theta, \forall l \mathfrak{N}^{*}\left(\alpha+\omega^{\mu} l, a+1\right), \\
& \forall l \mathfrak{A}^{*}\left(\alpha+\omega^{\mu} l, \mathfrak{t}\right), \Gamma \rightarrow \Theta
\end{aligned}
$$

are provable, accordingly, by Theorem 2 , we can see the provability of

$$
3(\beta, t), \Gamma \rightarrow \Theta \text {. }
$$

II) By the premises of the theorem, we can see that the sequents $\Gamma \rightarrow \Theta, \exists l \mathfrak{Q}^{*}\left(\alpha+\omega^{\mu} l, 1\right)$,

$\forall l \mathfrak{3}\left(\alpha+\omega^{\mu} l, a\right), \exists l \mathfrak{P}^{*}\left(\alpha+\omega^{\mu} l, a\right), \Gamma \rightarrow \Theta, \exists l \mathfrak{X}^{*}\left(\alpha+\omega^{\mu} l, a+1\right)$, $\exists l \mathfrak{A} *\left(\alpha+\omega^{\mu} l, \mathfrak{t}\right), \Gamma \rightarrow \Theta$

are provable, accordingly, by Theorem 2 , we can see the provability of 


$$
3(\beta, f), \Gamma \rightarrow \Theta
$$

q.e.d.

2. Number-theoretic formal system.

Number-theoretic formulae we shall define in the scope of the predicate logic of first order and in the usual way, by helps of numerals (as individual symbols), decidable predicates and the predicate symbol $Z$ (as predicate symbols), and calculable functions (as function symbols). As rules of inference we shall use ones for $L K$ and the following

Additional number-theoretic rules of inference.

$$
\begin{gathered}
\frac{Z(\mathrm{t}+1), \Gamma \rightarrow \Theta}{Z(\mathrm{t}), \Gamma \rightarrow \Theta} \\
\frac{\Gamma \rightarrow \Theta, \mathfrak{g}=\mathrm{t} \quad Z(\mathrm{t}), \Gamma \rightarrow \Theta}{Z(\mathfrak{s}), \Gamma \rightarrow \Theta} \\
\qquad \rightarrow \Theta, \mathfrak{A}(1) \quad Z(a), \mathfrak{A}(a), \Gamma \rightarrow \Theta, \mathfrak{A}(a+1) \quad \mathfrak{A}(\mathrm{t}), \Gamma \rightarrow \Theta \\
\hline Z(\mathrm{t}), \Gamma \rightarrow \Theta
\end{gathered}
$$

where $s$ and $t$ are arbitrary terms and the free variable $a$ of the last rule will never occur in $\Gamma, \Theta$ and $\mathfrak{A}(1)$. Each inference conforming the last rule is called 'mathematical induction', abbr. 'MI', and the formula $\mathfrak{A}(a)$ is called the induction formula.

As formal proofs we use ones in tree form whose uppermost sequents are arbitrary sequents of the form $\mathfrak{D} \rightarrow \mathfrak{D}$ or arbitrary sequents containing no logical symbols, provided that the latter sequents are effectively true in the usual interpretation. Then, the number-theoretic provability of a formula $\mathfrak{A}$ means the fact that there exists a formal proof of the sequent

$$
Z(1) \rightarrow \mathfrak{A} \text {. }
$$

Also on this number-theoretic formal system, the following cut-elimination theorem can be easily proved in almost the same way as in Gentzen's proof of the Hauptsatz on $L K$.

If a sequent is provable in the above-mentioned number-theoretic formal system, then the sequent is provable without cut in that formal system.

3. By 'restricted number theory' we shall mean the subsystem obtained from the above-mentioned number-theoretic formal system by adding the restriction on induction formulae:

The induction formula of each mathematical induction must be Z-positive or Z-negative.

A formula $\mathfrak{A}$ is said to be provable in the restricted number theory, if there exists a formal proof in the restricted number theory whose endsequent is

$$
Z(1) \rightarrow \mathfrak{A} \text {. }
$$

4. We shall prove our consistency theorem, in a generalized form as in the following

THEOREM 4. If a formula $\mathfrak{A}$ is provable in the restricted number theory, then there exists an ordinal number $r\left(<\omega^{\omega}\right)^{6)}$, and the formula $\mathfrak{I}^{*}$, which is

6) We assume that $\gamma$ is represented as the type containing no $n$-variable.

$$
-62-
$$


the result of substituting $\lambda z \mathfrak{Z}(0, z)$ for all positive ${ }^{7)} Z$ and $\lambda z \mathfrak{Z}(r, z)$ for all negative ${ }^{7} Z$ throughout $\mathfrak{A}$, is provable in the ramified theory, i.e.: the sequent $\rightarrow \mathfrak{A}^{*}$ is provable in the ramified theory.

COROLLARY. The restricted number theory is consistent, i.e.: the sequent

$$
Z(1) \rightarrow
$$

is unprovable in the restricted number theory.

PROOF OF THE COROLLARY. If the restricted number theory were inconsistent, then the formula $1=2$ should be provable in that theory, accordingly, by the above theorem, the sequent

$$
\rightarrow 1=2
$$

should be provable in the ramified theory. But it contradicts to the consistency of the ramified theory, q.e.d.

5. PROOF OF THEOREM 4.

Let $\mathbf{P}$ be a formal proof in the restricted number theory whose endsequent is

$$
Z(1) \rightarrow \mathfrak{A} \text {. }
$$

We can assume without loss of generality, by the cut-elimination theorem on the number-theoretic formal system, that $\mathbf{P}$ contains not cut.

For each MI (mathematical induction) in $\mathbf{P}$, we define the order of it, as follows :

1) The order of each uppermost MI is 0.

2) If the greatest order of all MI's which are above a given MI is $\mu$, then the order of the given MI is $\mu+1$.

Let the greatest order of MI in $\mathbf{P}$ be $\nu$. We substitute an expression of the form $\lambda z \mathcal{Z}\left(\alpha_{\mu}+\omega^{\mu} n_{\mu}, z\right)$ or $\lambda z \mathcal{Z}\left(\alpha_{\mu}+\omega^{\mu}\left(n_{\mu}+1\right), z\right)$ for each occurrence of $Z$ in $\mathbf{P}(\mu=0$, $1,2, \cdots, \nu)$, in the following manner, where

$$
n_{\nu}, n_{\nu-1}, \cdots, n_{1}, n_{0}
$$

are distinct free $n$-variables and $\alpha_{\mu}$ means the type

$$
\omega^{\nu} n_{\nu}+\omega^{\nu-1} n_{\nu-1}+\cdots+\omega^{\mu+1} n_{\mu+1},
$$

if $\mu<\nu$, or means 'empty', if $\mu=\nu$. When the order of MI

in $\mathbf{P}$ is $\mu$,

$$
\frac{\Gamma \rightarrow \Theta, \mathfrak{A}(1) \quad Z(a), \mathfrak{A}(a), \Gamma \rightarrow \Theta, \mathfrak{H}(a+1) \quad \mathfrak{A}(\mathrm{t}), \Gamma \rightarrow \Theta}{Z(\mathfrak{t}), \Gamma \rightarrow \Theta}
$$

1) all positive $Z$ in $\mathfrak{X}(1)$ and in $\mathfrak{A}(a+1)$, all negative $Z$ in $\mathfrak{A}(a)$ and in $\mathfrak{A}(t)$, and all their ancestors in $\mathbf{P}$ are substituted by $\lambda z \mathcal{Z}\left(\alpha_{\mu}+\omega^{\mu} n_{\mu}, z\right)$, and

2) all negative $Z$ in $\mathfrak{A}(1)$ and in $\mathfrak{H}(a+1)$, all positive $Z$ in $\mathfrak{A}(a)$ and in $\mathfrak{A}(\mathfrak{t})$, $Z$ of $Z(a)$, and all their ancestors are substituted by $\lambda z 3\left(\alpha_{\mu}+\omega^{\mu}\left(n_{\mu}+1\right), z\right)$. And we substitute $\lambda z \mathfrak{Z}(0, z)$ for all positive $Z$ in $\mathfrak{A}$ of the endsequent of $P$, and for all their ancestors, and substitute $\lambda z \mathbb{Z}\left(\omega^{\nu+1}, z\right)$ for all negative $Z$ in $\mathfrak{A}$ of the endsequent, for $Z$ of $Z(1)$ of the endsequent, and for all their ancestors.

7) An occurrence of a predicate symbol in a formula is said to be positive or negative, if the corresponding occurrence in the prenex disjunctive normal form of the formula is not preceded or is preceded by the negation symbol, respectively.

$$
-63-
$$


Now, let $\mathbf{P}^{*}$ mean the result obtained from $\mathbf{P}$ by the above-mentioned substitutions. Then the all uppermost sequents of $\mathbf{P}^{*}$ are provable in the ramified theory [cf. 1) of Theorem 1] and all inference-figures in $\mathbf{P}^{*}$ are correct in the sense of the ramified theory [cf. 3) and 4) of Theorem 1, and Theorem 3]. Especially, the lowermost sequent of $\mathbf{P}^{*}$ is

$$
3\left(\omega^{\nu+1}, 1\right) \rightarrow \mathfrak{U}^{*},
$$

where $\mathfrak{A}^{*}$ is the result of substituting $\lambda z \mathfrak{Z}(0, z)$ for all positive $Z$ and $\lambda z \mathfrak{Z}\left(\omega^{\nu+1}, z\right)$ for all negative $Z$ throughout $\mathfrak{A}$. Therefore, by the provability of the sequent

$$
\rightarrow \mathfrak{B}\left(\omega^{\nu+1}, 1\right)
$$

[cf. 2) of Theorem 1], we can show that the sequent

$$
\rightarrow \mathfrak{U} *
$$

is provable in the ramified theory, q.e.d.

Our consistency proof has been completed in the above.

(Received, Dec. 22, 1961)

\section{References}

[1] G. Gentzen, Untersuchungen über das logische Schließen. Math. Z., 39, 176-210, 405431 (1935).

[2] S. C. Kleene, Introduction to metamathematics. Amsterdam/Groningen, 1952.

[3] K. Ono, Logische Untersuchungen über die Grundlagen der Mathematik. J. Fac. Sci. Imp. Univ. Tokyo, Sec. I, 3, 328-389 (1938). 